\title{
A methodology for assessing human mitral leaflet curvature using real-time 3-dimensional echocardiography
}

Liam P. Ryan, MD, ${ }^{\text {a } B e n j a m i n ~ M . ~ J a c k s o n, ~ M D, ~}{ }^{\text {a }}$ Thomas J. Eperjesi, BS, ${ }^{\text {a }}$ Theodore J. Plappert, CVT, Martin St John-Sutton, MBBS, FRCP, ${ }^{b}$ Robert C. Gorman, MD, and Joseph H. Gorman, III, MD ${ }^{a}$

From the Harrison Department of Surgical Research $^{\mathrm{a}}$ and the Department of Medicine, ${ }^{\mathrm{b}}$ University of Pennsylvania School of Medicine, Philadelphia, $\mathrm{Pa}$.

Supported by National Institutes of Health (Bethesda, Md) grants HL63954 (RCG), HL73021, and HL76560 (JHG) and an American Heart Association (Glen Allen, Va) Post-Doctoral Fellowship 0625455U (LPR).

Received for publication Oct 25, 2007; revisions received Dec 20, 2007; accepted for publication Feb 3, 2008.

Address for reprints: Joseph H. Gorman, III, MD, Department of Surgery, University of Pennsylvania, Glenolden Research Laboratory, $500 \mathrm{~S}$ Ridgeway Ave, 2nd Floor, Room 249, Glenolden, PA 19036 (E-mail: gormanj@uphs.upenn.edu).

J Thorac Cardiovasc Surg 2008;136:726-34 $0022-5223 / \$ 34.00$

Copyright (C) 2008 by The American Association for Thoracic Surgery

doi:10.1016/j.jtcvs.2008.02.073
Objectives: Using 3-dimensional echocardiography in conjunction with novel geometric modeling and rendering techniques, we have developed a high-resolution, quantitative, 3-dimensional methodology for imaging the human mitral valve. Leaflet and annular geometry are important determinants of mitral valve stress. Repair techniques that optimize valvular geometry will reduce stress and potentially increase repair durability. The development of such procedures will require image-processing methodologies that provide a quantitative description of 3-dimensional valvular geometry.

Methods: Ten healthy adult subjects underwent mitral valve imaging with real-time 3-dimensional echocardiography. By using specially designed image analysis software, multiple valvular geometric parameters, including 2- and 3-dimensional leaflet curvature, leaflet surface area, annular height, intercommissural width, septolateral annular diameter, and annular area were determined for each subject. Image-rendering techniques that allow for the clear and concise presentation of this detailed information are also presented.

Results: Although 3-dimensional annular and leaflet geometry were found to be highly conserved between healthy human subjects in general, substantial intrasubject and intersubject regional geometric heterogeneity was observed in the midposterior leaflet, the region most commonly involved in leaflet flail in subjects with myxomatous disease.

Conclusions: The image-processing and graphic-rendering techniques that we have developed can be used to provide a complete description of 3-dimensional mitral valve geometry in human subjects. Widespread application of these techniques to healthy subjects and patients with mitral valve disease will provide insight into the geometric basis of both valvular pathology and repair durability.

$\mathrm{L}$ onger-term follow-up demonstrates that between $10 \%$ and $16 \%$ of patients undergoing mitral repair for myxomatous disease will require reoperation for severe mitral regurgitation (MR) within 10 years. ${ }^{1-4}$ Even more concerning are the recent reports of an unexpectedly high incidence of recurrent moderate MR after mitral valve repair. Four recent studies from experienced centers indicate that the recurrence of $2+$ or greater MR is between $2 \%$ and $4 \%$ per year. ${ }^{5-8}$ Considering patients with even mild residual/recurrent MR after valve repair have decreased exercise tolerance and longevity compared with patients with competent repairs, ${ }^{9}$ there is a need to improve current repair techniques. This is particularly relevant because an increasing number of asymptomatic patients are being referred for valve repair. ${ }^{10-12}$

A significant number of repair failures result from chordal, leaflet, and suture-line disruption, which suggests that mechanical leaflet stress plays a significant role in this process. ${ }^{13}$ It is recognized that leaflet curvature is an important determinant of valve 


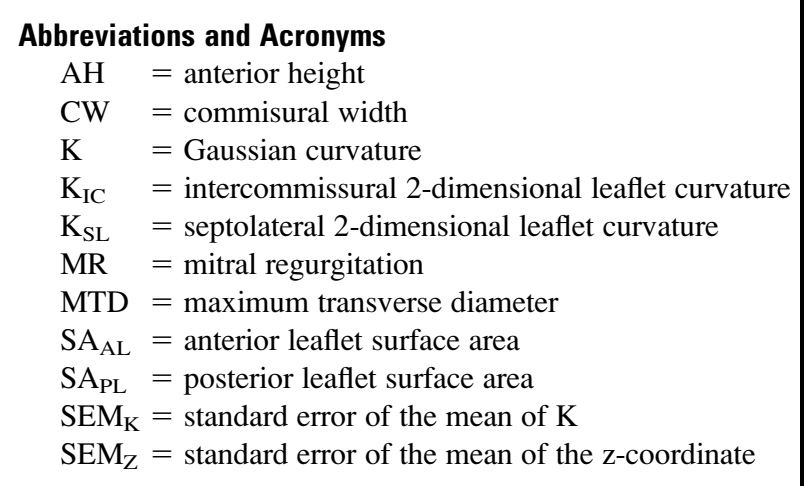

stress. ${ }^{14}$ As such, repair devices and techniques that maximize leaflet curvature might reduce valvular stress and increase repair durability. To prove this concept, it is necessary to develop clinically applicable quantitative imaging techniques that allow both the assessment of normal leaflet geometry and the effect of repair techniques on leaflet geometry. Only through a more thorough understanding of normal 3-dimensional mitral valve geometry can reconstructive strategies realistically be directed toward restoration of this geometry.

Real-time 3-dimensional echocardiography, which is noninvasive, relatively inexpensive, and widely available, is uniquely applicable to this task because it allows for both high-resolution 3-dimensional rendering of the mitral valve ${ }^{15}$ and the calculation of multiple geometric parameters, including spatially resolved 3-dimensional Gaussian curvature on the leaflet surfaces.

In this study we describe several novel echocardiographically based techniques for quantifying mitral valve geometry and apply these techniques to the characterization of normal human mitral valve geometry. Additionally, we introduce the concept of regional curvature heterogeneity, which has previously been proposed as a marker of complex stress distribution patterns, ${ }^{16}$ to the quantitative description of the mitral valve.

\section{Materials and Methods \\ Image Acquisition}

The study was approved by the University of Pennsylvania Institutional Review Board. Each subject provided informed consent. Transthoracic echocardiographic analysis was performed in 10 healthy human adult male patients. Four full-volume data sets were acquired with a Sonos 7500 (Philips, Andover, Mass) equipped with a 2- to 4-MHz X4 handheld matrix transducer for each subject. Electrocardiographically gated images were acquired over 8 cardiac cycles. Each full-volume data set was then exported to a Cardio-View (Tomtec Imaging Systems, Munich, Germany) software workstation for image analysis. The highest-quality data set was selected for each subject by an experienced echocardiographer. The remaining 3 data sets were excluded from the current study.
Calculations were performed with Matlab (The Mathworks, Inc, Natick, Mass) software.

\section{Image Analysis}

Cardio-View allows for the interactive manipulation, including rotation, translation, surface rendering, and measurement, of fully 3-dimensional ultrasonographic data sets (4-dimensional data). All analysis was performed at midsystole to minimize load variability. The plane of the mitral valve orifice was rotated into a short-axis view. The geometric center was then translated to the intersection of the 2 corresponding long-axis planes, which then corresponded to the intercommissural and septolateral axes of the mitral valve orifice. A rotational template consisting of 18 long-axis cross-sectional planes separated by $10^{\circ}$ increments was superimposed on the 3-dimensional echocardiogram (Figure 1, A). The 2 annular points intersecting each of the 18 long-axis rotational planes were then identified by means of orthogonal visualization of each plane; the 2 points were marked interactively (Figure 1,B). Measurement planes were then marked at fixed 2-mm intervals along the entire length of the intercommissural axis (Figure 1, C).

Free-hand curves, each consisting of between 5 and 40 data points, connecting the juxtaposed anterior and posterior annulus across the atrial surfaces of the leaflets (Figure 1, D) were constructed in each long-axis cross-section, resulting in a 500- to 1000-point data set for each valve (Figure 2). Each data point fit within a given measurement plane was then assigned to the anterior mitral leaflet, the posterior mitral leaflet, or the coaptation point.

\section{Calculation of Annular Geometry}

Geometric modeling and analysis of each annular data set were performed by using a series of Matlab algorithms. The center of gravity of the data set was translated to the origin. The least-squares plane of the 3-dimensional data set was then calculated by means of orthogonal distance regression, and the annular model was rotated such that this mitral valve orifice plane was aligned with the $x-y$ plane. The data set was then rotated around the z-axis, such that the $\mathrm{x}$-axis bisected the midanterior and midposterior annulus. Under these geometric conditions, the z-coordinate of each annular point was therefore equal to its distance to the best-fit plane of the mitral valve. The annular height of a given data set was defined as $Z_{\max }-Z_{\min }$. The septolateral diameter for a given data set was defined as the distance, in 3-dimensional space, separating the 2 data points located along the x-axis: the midanterior and midposterior annuli. The intercommissural width for a given data set was defined as the distance, in 3-dimensional space, separating the 2 data points with the lowest values of the $\mathrm{z}$-coordinate: the anterior and posterior commissures. The maximum transverse diameter (MTD) was defined as the distance, in 3-dimensional space, separating the 2 annular data points with maximum and minimum y coordinates (eg, the widest point of the mitral annulus). The annular height (AH)/commissural width $(\mathrm{CW})$ ratio for a given annular data set was defined as follows: $[A H \div C W] \times 100 \%$. Similarly, the AH/MTD ratio was defined as follows: $[A H \div M T D] \times 100 \%$. The annular aspect ratio was defined as the MTD/septolateral diameter ratio. Finally, the mitral annular area was defined as the area enclosed by the 2-dimensional projection of a given annular data set onto its corresponding least-squares plane. 

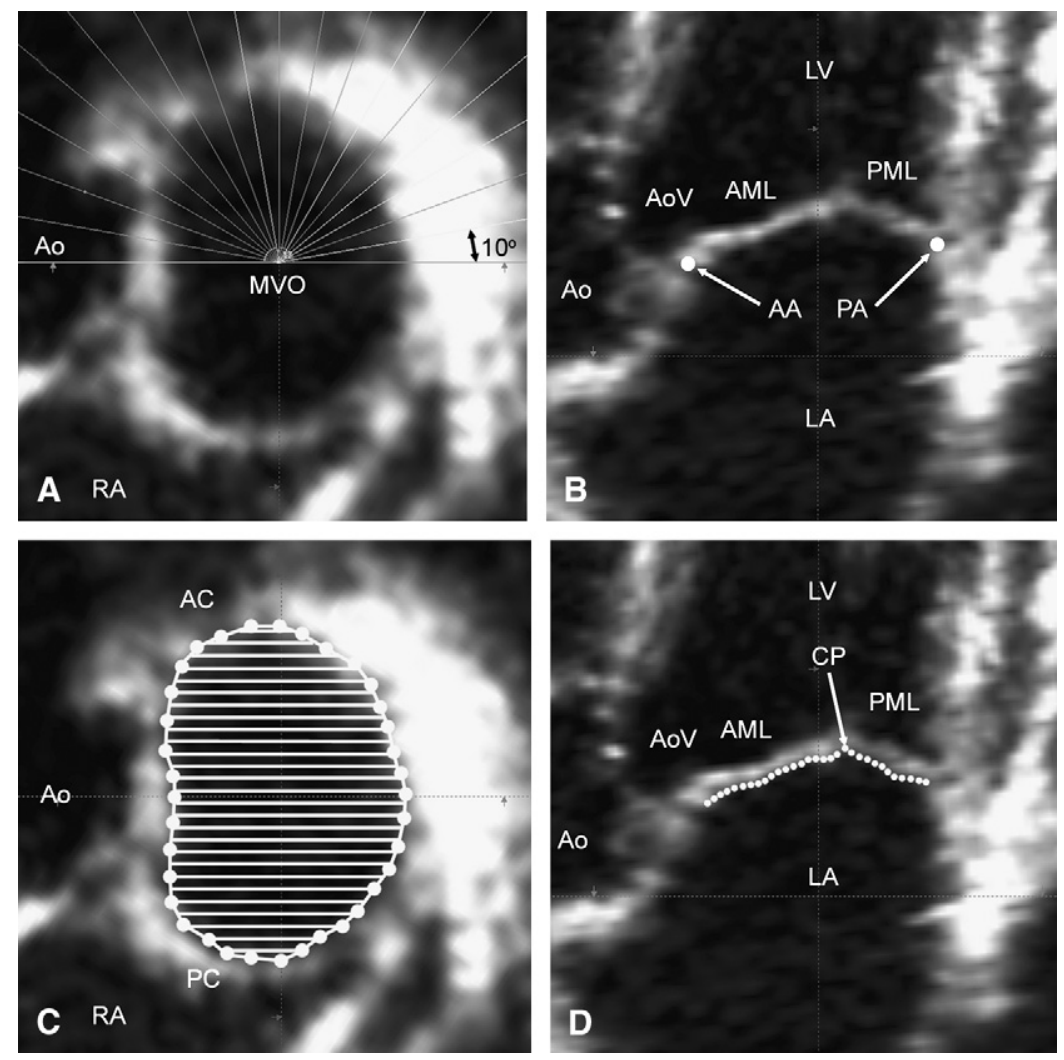

Figure 1. A, View of the mitral valve, where the selected short-axis plane coincides with the plane of the mitral valve. The aorta $(\mathrm{Ao})$ and mitral valve orifice (MVO) are indicated. A rotational template consisting of 18 long-axis planes evenly spaced at $10^{\circ}$ increments and centered at the geometric center of the mitral valve has been constructed. B, Single long-axis view $\left(0^{\circ}\right.$ on the rotational template of panel $A$ ) of the heart. The left ventricle $(L V)$, anterior $(A M L)$ and posterior (PML) mitral leaflets, left atrium (LA), aortic valve $(A o V)$, and aorta $(A o)$ have been illustrated. Anterior $(A A)$ and posterior $(P A)$ annular points have been marked. Note that in this orientation the negative z-axis (for purposes or annular height calculations) extends toward the apex, whereas the positive z-axis extends toward the left atrium. All mitral annular data points are shown in panel C, as are each of the septolateral measurement planes, which are spaced at 1-mm intervals along the intercommissural axis. The anterior commissure $(A C)$, posterior commissure (PC), aorta $(A o)$, and right atrium $(R A)$ are labeled in this view. As shown in panel $D$, in each of the septolateral measurement planes, between 5 and $\mathbf{4 0}$ individual points (depending on the septolateral diameter of the mitral valve at the position of a given measurement plane) on the atrial surface of the mitral valve are manually marked. The position of each point is recorded as anterior mitral leaflet (AML), coaptation point (CP), or posterior mitral leaflet (PML). Ao, Aorta; $A o V$, aortic valve; $L V$, left ventricle; $L A$, left atrium.
Calculation of Leaflet Surface Area and Leaflet Curvature

Each point along the annulus was merged with the leaflet data set, and the coaptation points were added to enforce leaflet coaptation. The center of gravity of each mitral valve data set was translated to the origin. The least-squares plane of the 3-dimensional data set was then calculated by means of orthogonal distance regression. The data set was then rotated so that the least-squares plane of the mitral valve corresponded to the xy-plane and so that the meridianal septolateral axis corresponded to the $\mathrm{x}$-axis. Under these geometric conditions, the intercommissural axis lay parallel to the $y$-axis.

Separate meshed grids were then created for the anterior leaflet and posterior leaflet data sets. Smoothing splines were constructed separately for anterior and posterior leaflets by using the Matlab spline TPAPS function. The smoothing parameter, which varies between 0 for a least-squares approximation and 1 for a thin-plate spline interpolant, was assigned automatically for each data set by the Matlab software. The splined z-coordinates at each mesh point
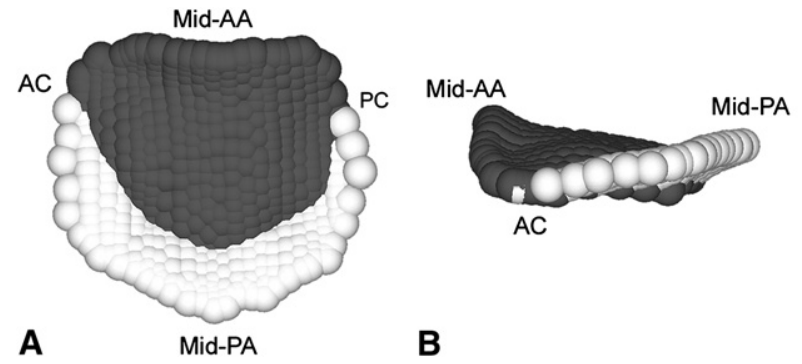

B

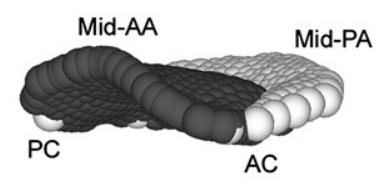

C

Figure 2. A typical 600-point 3-dimensional data set for a normal human mitral valve is shown in 3 views: transvalvular (A), intercommissural (B), and oblique (C). Data points on the anterior leaflet are shaded gray, whereas those on the posterior leaflet are shaded white. The midanterior annulus (mid$A A)$, midposterior annulus (mid-PA), anterior commissure $(A C)$, and posterior commissure ( $P C$, if visible) are indicated for purposes of orientation. 
of the rectangular mesh grid were calculated separately over each leaflet.

The area of each triangle described by 3 adjacent mesh points was then calculated continuously across the leaflet meshed grids. The anterior leaflet surface area $\left(\mathrm{SA}_{\mathrm{AL}}\right)$ was then defined as the sum of triangular areas across the anterior leaflet, whereas the posterior leaflet surface area $\left(\mathrm{SA}_{\mathrm{PL}}\right)$ was defined as the sum of triangular areas across the posterior leaflet. The total surface area was then defined as the sum of $\mathrm{SA}_{\mathrm{AL}}$ and $\mathrm{SA} \mathrm{PL}_{\mathrm{PL}}$. The $\mathrm{SA}_{\mathrm{AL}} / \mathrm{SA}_{\mathrm{PL}}$ ratio and total leaflet surface area/annular area ratio were subsequently calculated for each subject.

The Gaussian curvature (K) $K=\frac{\operatorname{det}\left(x_{u u} x_{u} x_{v}\right) \operatorname{det}\left(x_{v v} x_{u} x_{v}\right)-\left[\operatorname{det}\left(x_{u v} x_{u} x_{v}\right)\right]^{2}}{\left[\left|x_{u}\right|^{2}\left|x_{v}\right|^{2}-\left(x_{u} x_{v}\right)^{2}\right]^{2}}$ of the spline fits was then calculated for every mesh point on both grids.

Septolateral and intercommissural 2-dimensional leaflet curvatures $\left(\mathrm{K}_{\mathrm{SL}}\right.$ and $\left.\mathrm{K}_{\mathrm{IC}}\right)$ were then calculated as follows. Let $\mathrm{a}, \mathrm{b}$, and $\mathrm{c}$ be the distances between each of 3 sequential mesh points along either the $\mathrm{x}$-axis (septolateral) or $\mathrm{y}$-axis (intercommissural). The 2-dimensional curvature along the selected axis at a given mesh point is then defined as follows:

$$
K_{S L} \text { or } K_{I C}=\frac{\sqrt{(a+b+c) \cdot(b+c-a) \cdot(c+a-b) \cdot(a+b-c)}}{a \cdot b \cdot c}
$$

Both $\mathrm{K}_{\mathrm{SL}}$ and $\mathrm{K}_{\mathrm{IC}}$ were calculated for every mesh point on both anterior and posterior leaflet grids.

\section{Description of Regional Gaussian Curvature Heterogeneity}

The standard error of the mean of $\mathrm{K}\left(\mathrm{SEM}_{\mathrm{K}}\right)$ over a 7-point $\times 7$ point region $\left(0.49 \mathrm{~mm}^{2}\right)$ was calculated and assigned to the mesh point at the center of the $7 \times 7$-point region to quantify leaflet geometric heterogeneity (eg, geometric variability) for a given subject. This calculation was performed continuously for overlapping regions across the entire valve surface.

\section{Construction of Hybrid 3-Dimensional Renderings}

A 3-dimensional hybrid rendering was constructed for all subjects $(n=10)$ to facilitate the presentation of quantitative geometric data.
The hybrid anterior and posterior leaflets were modeled independently. The individual geometric centers of each anterior and posterior leaflet mesh within a given cohort were translated to their averaged geometric centers, scaled to their averaged area in 2-dimensional (xy) space, and then overlapped. Those areas of the overlapped individual leaflet meshes in which all data sets, or all data sets except one, coincided were included in the hybrid mesh. All other points were excluded. The z-coordinate at each point along the hybrid anterior and posterior leaflet meshes was determined by using the arithmetic mean of the vertically compiled z-coordinates of the individual overlapped models. Finally, the point in 2-dimensional (xz) space at which anterior and posterior leaflet meshes intersected was defined for each mesh interval along the intercommissural (y) axis. The resultant series of coaptation points was then defined as the line of leaflet coaptation for the 3-dimensional rendering.

Four distinct shading techniques, in which interpolated color contouring at a given mesh point was determined by $\mathrm{K}_{\mathrm{SL}}$ (Figure 3), $\mathrm{K}_{\mathrm{IC}}$ (Figure 4), K (Figure 5), and $\mathrm{SEM}_{\mathrm{K}}$ (Figure 6), were then applied to each hybrid rendering to facilitate visualization of each of these geometric indexes in 3-dimensional space. In each case color intensity was determined by the mean value of the given index for the 10 vertically compiled mesh points.

\section{Description of Intersubject Leaflet Geometric Heterogeneity}

The standard error of the mean of the $\mathrm{z}$-coordinate $\left(\mathrm{SEM}_{\mathrm{Z}}\right)$ was calculated for the 10 vertically compiled mesh points of the individual overlapped models (see above) across the entire mitral valve surface to quantify leaflet geometric heterogeneity (eg, geometric variability) for the 10-subject cohort. A 3-dimensional hybrid rendering was then constructed in which color contouring was determined based on $\mathrm{SEM}_{\mathrm{Z}}$ at a given mesh point (Figure 7).

\section{Results}

On average, image analysis and all subsequent calculations required approximately 90 minutes to complete for each subject.

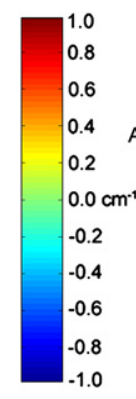

A
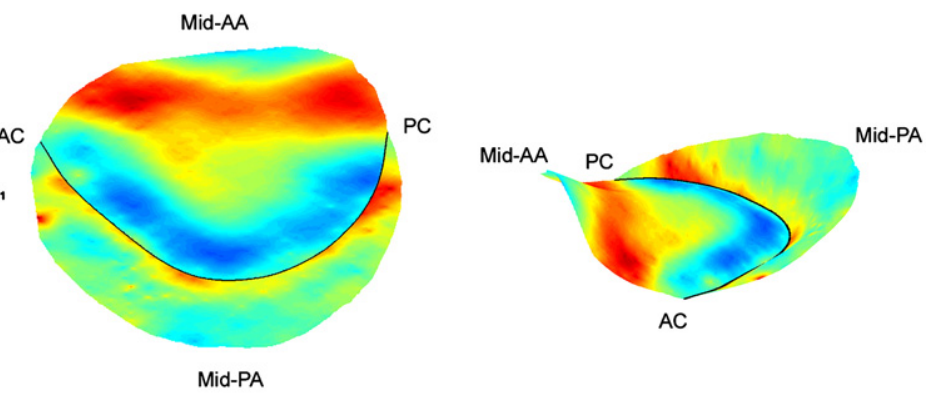

B
Figure 3. Transvalvular (A) and oblique (B) views of a hybrid 3-dimensional rendering reflecting the average 3-dimensional valvular geometry of the 10 -subject cohort in which color contouring is determined by the 2-dimensional curvature of the leaflets across the septolateral axis of the mitral valve. The midanterior annulus (mid-AA), midposterior annulus (mid-PA), anterior commissure $(A C)$, and posterior commissure (PC) have been labeled in each view. Note the prominent compound curvature of the anterior leaflet in which the concavity across the leaflet belly is atrially directed (dark red) and note that adjacent to the line of coaptation is apically directed (dark blue). Conversely, curvature across the posterior leaflet is predominantly simple, with apically directed concavity (light blue). 


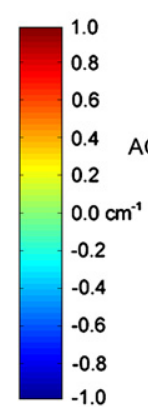

A

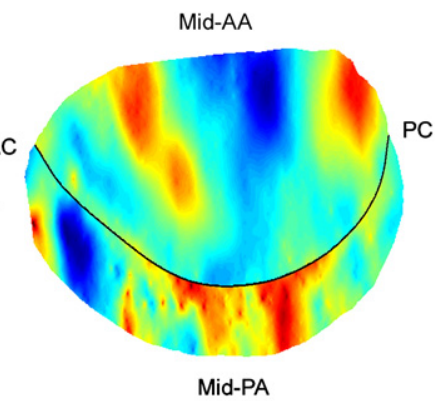

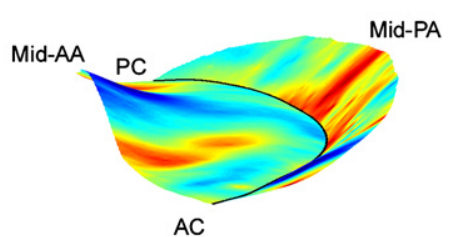

B
Figure 4. Transvalvular (A) and oblique (B) views of a hybrid 3-dimensional rendering reflecting the average 3-dimensional valvular geometry of the 10 -subject cohort in which color contouring is determined based on the 2-dimensional curvature of the leaflets across the intercommissural axis of the valve. The midanterior annulus (mid-AA), midposterior annulus (mid-PA), anterior commissure $(A C)$, and posterior commissure (PC) have been labeled in each view. Note the alternating pattern of curvature orientation both adjacent to the anterior annulus and across the belly of the anterior leaflet: the concavity is apically oriented (dark blue) along the meridian of the leaflet (A2) and atrially oriented (yellow and dark red) along the medial (A3) and lateral (A1) surfaces of the leaflet. The intercommissural axis alternates continuously across the intercommissural axis of the posterior, although this pattern is most prominent within P2.

\section{Annular Geometry and Leaflet Surface Area}

Measurements of annular shape and leaflet surface area are presented in Table 1 as means \pm standard error of the mean.

\section{Leaflet Curvature}

Patterns of 2-dimensional and 3-dimensional leaflet curvature for the 10-subject cohort are shown in Figures 3 through 5. As discussed in detail above, each rendering reflects the average 3-dimensional geometry of the 10-subject cohort. Color contouring is determined by the mean value of $\mathrm{K}_{\mathrm{SL}}$ (Figure 3), $\mathrm{K}_{\mathrm{IC}}$ (Figure 4), or K (Figure 5) for the 10 vertically compiled mesh points at each mesh point within the hybrid rendering.

On inspection of Figure 3, which shows patterns of 2-dimensional septolateral curvature along the leaflets, 2 pronounced geometric features emerge. Most notably, $\mathrm{K}_{\mathrm{SL}}$ across the anterior leaflet is uniformly compound, with highly negative (apically oriented concavity) curvature immediately adjacent to the midanterior annulus, highly positive (atrially oriented concavity) curvature within the leaflet belly, and then highly negative (apically oriented concavity) curvature adjacent to the line of coaptation. Conversely, $\mathrm{K}_{\mathrm{SL}}$ across the posterior leaflet is predominantly simple, with negative (apically oriented concavity) curvature both adjacent to the posterior annulus and within the leaflet belly and a relatively flat surface $\left(\mathrm{K}_{\mathrm{SL}}\right.$ between 0.0 and $\left.0.2 \mathrm{~cm}^{-1}\right)$ adjacent to the line of coaptation, although there are scattered foci of highly positive curvature (atrially oriented concavity) within this region.

Similarly, 2 pronounced patterns of 2-dimensional intercommissural curvature emerge on inspection of Figure 4. Most notable is the manner in which $\mathrm{K}_{\mathrm{IC}}$ alternates across the intercommissural axis of the anterior leaflet, with highly

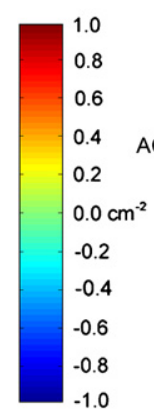

A

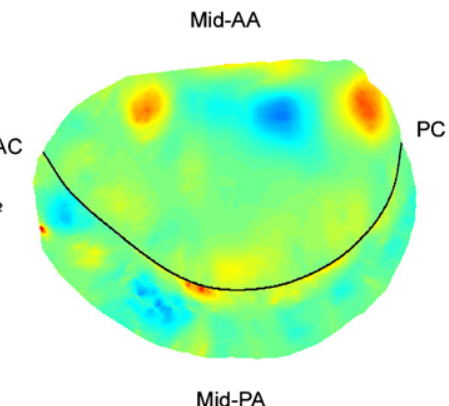

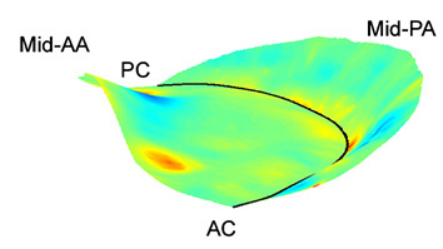

B 


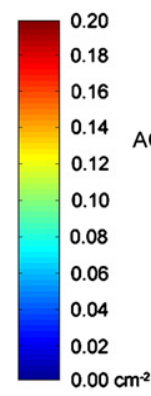

A

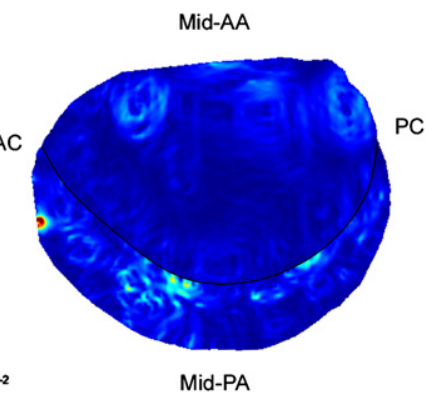

Mid-AA

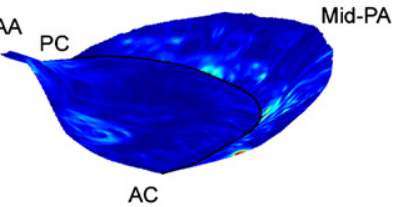

B
Figure 6. Transvalvular (A) and oblique (B) views of a hybrid 3-dimensional rendering reflecting the average 3-dimensional valvular geometry of the 10 -subject cohort in which color contouring is determined by the regional standard error of the mean of Gaussian curvature across the individual leaflet surfaces. Thus the intensity of the color contouring reflects the degree of intrasubject regional geometric heterogeneity. There are 2 prominent foci of curvature heterogeneity adjacent to the line of coaptation within $\mathrm{P2}$ and a third focus adjacent to the posterior annulus within P1. Otherwise, curvature is highly homogeneous across the mitral valve. The midanterior annulus (mid-AA), midposterior annulus (mid-PA), anterior commissure $(A C)$, and posterior commissure (PC) have been labeled in each view. positive (atrially oriented concavity) curvature within A1, highly negative (apically oriented concavity) curvature centered on the A2 meridian, and then highly positive (atrially oriented concavity) curvature again on A3. It is interesting to note that this pattern of curvature mirrors the geometry of the anterior annular saddle horn. Although $\mathrm{K}_{\mathrm{IC}}$ also alternates across the intercommissural axis of the posterior leaflet, the spatial distribution of positive and negative curvature is reversed from that observed across the anterior leaflet: $\mathrm{K}_{\mathrm{IC}}$ is highly negative (apically oriented concavity) within P1, predominantly positive (atrially oriented concavity) within $\mathrm{P} 2$, and then negative (apically oriented concavity) within P3. There are also 2 distinct foci of highly positive (atrially oriented concavity) $\mathrm{K}_{\mathrm{IC}}$ within $\mathrm{P} 2$ separated by a relatively flat region along the $\mathrm{P} 2$ meridian.

Regional patterns of 3-dimensional leaflet deformation are shown in Figure 5. Although Gaussian curvature (K) was calinition of $\mathrm{K}$ is as follows: ${ }^{K=k 1 \times k 2}$, where $\mathrm{k} 1$ and $\mathrm{k} 2$ are principal (major and minor) curvatures that measure the maximum and minimum 2-dimensional curvature at a given point on a regular surface. Values of $\mathrm{K}$ less than 0 (either $\mathrm{k} 1$ or $\mathrm{k} 2$ is negative) indicate the presence of a hyperbolic surface, whereas values of $\mathrm{K}$ greater than $0(\mathrm{k} 1$ and $\mathrm{k} 2$ are either both positive or both negative) indicate an elliptical surface. Although $\mathrm{K}$ does indicate the relative orientations (signs) of the principal curvatures at a given point in space, it does not indicate the orientation of the surface within a 3-dimensional frame of reference. Although this can be readily appreciated on examination of the 3-dimensional model, it is difficult to appreciate in a 2-dimensional projection (Figure 3). However, $\mathrm{K}_{\mathrm{SL}}$ and $\mathrm{K}_{\mathrm{IC}}$ have been calculated for every mesh point as well, and although $\mathrm{K}_{\mathrm{SL}}$ and $\mathrm{K}_{\mathrm{IC}}$ do not necessarily coincide with $\mathrm{k} 1$ and $\mathrm{k} 2$, they do allow one to relate 2-dimensional culated by means of orthogonal gradients, an alternative def-

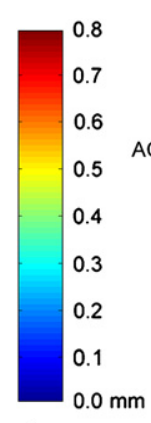

A

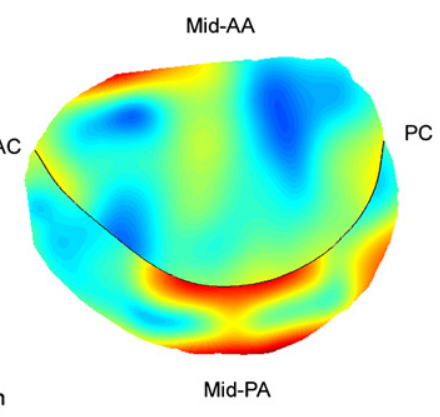

Mid-AA

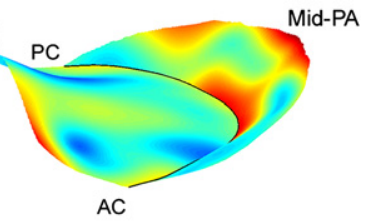

B
Figure 7. Transvalvular (A) and oblique (B) views of a hybrid 3-dimensional rendering reflecting the average 3-dimensional valvular geometry of the 10 -subject cohort in which color contouring is determined based on the standard error of the mean of the z-coordinates of the 10 vertically compiled mesh points at each mesh point across the mitral leaflets. Thus the intensity of color contouring reflects the degree of intersubject regional geometric heterogeneity. Across the anterior leaflet, there is a single focus of intersubject geometric heterogeneity (dark red) adjacent to the anterior annulus within A1 and A2: there is otherwise a high degree of geometric homogeneity between subjects across the anterior leaflet. Conversely, there is a high degree of intersubject geometric heterogeneity within P2 and P3. The midanterior annulus (mid-AA), midposterior annulus (mid-PA), anterior commissure (AC), and posterior commissure $(P C)$ have been labeled in each view. 
TABLE 1. Annular and leaflet geometry

\begin{tabular}{|c|c|}
\hline & Mean \pm SEM \\
\hline $\mathrm{AH}(\mathrm{mm})$ & $8.9 \pm 0.6$ \\
\hline CW (mm) & $33.6 \pm 1.9$ \\
\hline AHCWR (\%) & $26.4 \pm 1.0$ \\
\hline MTD (mm) & $41.5 \pm 0.0$ \\
\hline AHMTDR (\%) & $21.4 \pm 1.2$ \\
\hline $\operatorname{MAA}\left(\mathrm{mm}^{2}\right)$ & $1104 \pm 51$ \\
\hline $\mathrm{SL}(\mathrm{mm})$ & $32.2 \pm 0.7$ \\
\hline AAR & $1.3 \pm 0.0$ \\
\hline$S A_{A L}\left(m^{2}\right)$ & $862 \pm 55$ \\
\hline$S A_{P L}\left(m^{2}\right)$ & $437 \pm 31$ \\
\hline $\mathrm{SA}_{\text {Total }}\left(\mathrm{mm}^{2}\right)$ & $1298 \pm 80$ \\
\hline$S A_{A L} / S A_{P L}$ & $2.0 \pm 0.1$ \\
\hline$S A_{\text {Total }} / \mathrm{MAA}$ & $1.2 \pm 0.0$ \\
\hline
\end{tabular}

$S E M$, Standard error of the mean; $A H$, annular height; $C W$, intercommissural width; AHCWR, annular height/commissural width ratio; MTD, maximum transverse diameter; $A H M T D R$, annular height/maximum transverse diameter ratio; $M A A$, mitral annular area; $S L$, septolateral diameter; $A A R$, annular aspect ratio (MTD/SL); $S A_{A L}$, anterior leaflet surface area; $S A_{P L}$, posterior leaflet surface area; $S A_{\text {Total, }}$ total leaflet surface area; $S A_{A L} / S A_{P L}$, anterior/posterior leaflet surface area ratio; $S A_{T o t a} / M A A$, leaflet surface area/ mitral annular area ratio.

and 3-dimensional curvature at any point along the rendered surface. There is a prominent hyperbolic region adjacent to the midanterior annulus that reflects the intersection of positive (atrially oriented concavity) septolateral curvature and negative (apically oriented concavity) intercommissural curvature in this region. Additionally, there are prominent elliptical regions on both sides of this central hyperbolic surface, one adjacent to the posterior commissure and one adjacent to the anterior annulus within A1, that reflect the intersection of highly positive (atrially oriented concavity) septolateral and intercommissural in this region. Although there are 2 prominent hyperbolic foci ( $\mathrm{P} 1$ and $\mathrm{P} 2$ ) along the posterior leaflet surface, they do not appear to represent prominent 2-dimensional features, suggesting that $\mathrm{K}_{\mathrm{SL}}$ and $\mathrm{K}_{\mathrm{IC}}$ do not approximate $\mathrm{k} 1$ and $\mathrm{k} 2$ at these points on the mitral valve surface. Gaussian curvature across the remainder of the mitral valve surface exists over a relatively narrow range $(-0.2$ to $0.2 \mathrm{~cm}^{-2}$ ), indicating that although there is substantial regional variation in $\mathrm{K}_{\mathrm{SL}}$ and $\mathrm{K}_{\mathrm{IC}}$, 3-dimensional curvature is nonetheless relatively homogeneous.

\section{Leaflet Gaussian Curvature Heterogeneity}

The regional geometric variability of K is shown in Figure 6 . In this rendering color intensity reflects the standard error of the mean of $\mathrm{K}$ within a very small region $\left(0.49 \mathrm{~mm}^{2}\right)$ around a central mesh point $\left(\mathrm{SEM}_{\mathrm{K}}\right)$. Interestingly, the only foci of high heterogeneity $\left(\mathrm{SEM}_{\mathrm{K}}>0.08 \mathrm{~cm}^{-2}\right)$ occur across the posterior leaflet (there is a single focus of heterogeneity along the posterior annulus in P1 and 2 separate foci in P2), indicating spatially dense patterns of geometric variation within these regions. There is otherwise minimal $\left(\mathrm{SEM}_{\mathrm{K}}<0.04\right.$ $\mathrm{cm}^{-2}$ ) intrasubject regional geometric heterogeneity.

\section{Intersubject Regional Geometric Heterogeneity}

The variability of 3-dimensional geometry between subjects is shown in Figure 7. In this rendering color intensity reflects the standard error of the mean of the vertically compiled z-coordinates of the 10-subject cohort at each overlapping mesh point $\left(\mathrm{SEM}_{\mathrm{Z}}\right)$. Again, geometric heterogeneity is most prominent across the posterior leaflet surface: there are 2 large confluent foci of high intersubject heterogeneity $\left(\mathrm{SEM}_{\mathrm{Z}}>0.5\right.$ $\mathrm{mm}$ ) in $\mathrm{P} 2$ and an isolated focus in $\mathrm{P} 3$. There is a separate focus of high intersubject heterogeneity along the anterior annulus within $\mathrm{A} 1$ and $\mathrm{A} 2$. There are 3 additional foci of moderate $\left(\mathrm{SEM}_{\mathrm{Z}}\right.$ between 0.3 and $\left.0.5 \mathrm{~mm}\right)$ heterogeneity across the anterior leaflet, within the belly of $\mathrm{A} 2$, and adjacent to the line of coaptation in both A1 and A3. There is otherwise minimal intersubject regional geometric heterogeneity.

\section{Discussion}

The methodology presented provides a complete and quantitative description of normal mitral valve geometry. Geometricmodeling and graphic-rendering techniques have been used to characterize the orientation and magnitude of both orthogonally oriented 2-dimensional septolateral and intercommissural curvatures and 3-dimensional Gaussian curvature across the entire valve. The application of this methodology to 10 healthy human subjects has described mitral valve geometry in greater detail and complexity than ever before.

Although prior finite element models have improved our understanding of mitral valve loading patterns, each assumes passive billowing of the leaflets toward the atrium during systole. ${ }^{17-19}$ These assumptions have, in most but not all cases, been drawn from observing the valve in its unloaded state during postmortem or intraoperative examination. The previously unappreciated complexity of leaflet geometry described in this study is likely due to the intricate and multifarious interplay between the mitral annulus, mitral leaflets, chordae tendinae, contracting ventricle, and pressure loading. Incorporation of these quantitative anatomic models might allow for more accurate finite element modeling of the mitral valve. Such composite analysis might both improve our understanding of valvular physiology and potentially elucidate geometric predictors of mitral valve repair efficacy and durability.

It is possible that failure to restore normal valvular geometry might contribute to the chordal, leaflet, and suture-line disruption that frequently underlies repair failure in patients with degenerative mitral valve disease. ${ }^{13}$ Therefore we believe that a more quantitative understanding of 3-dimensional leaflet shape is essential to the ongoing improvement of clinical outcomes in this patient population. The work reported here is an important first step toward this goal. 
Although the mean leaflet surface area/mitral annular area ratio of 1.2 that we report is lower than previously published values, which range from 1.76 to $1.90,{ }^{20,21}$ this is readily explained by 2 distinct phenomena. First, these prior measurements were performed postmortem and are likely overestimates caused by fixation artifacts. Second, our calculated leaflet surfaces areas do not include the surface of coaptation and, by necessity, represent underestimates. Nonetheless, the technique that we describe allows for the quantitative characterization of leaflet redundancy in fully loaded valves in vivo, excluding the surface of coaptation.

Our results confirm those of previous studies involving invasive imaging techniques that have documented compound curvature along the septolateral meridian of the anterior leaflet and hyperbolic surface geometry within the belly of the midanterior (A2) leaflet zone. ${ }^{22-24}$ Furthermore, our findings indicate that compound curvature exists across the entire anterior leaflet. Additionally, our findings confirm that septolateral curvature across the posterior is predominantly simple. ${ }^{22,23}$

Although the saddle shape of the mitral annulus has been well documented, ${ }^{25,26}$ our description of $\mathrm{K}_{\mathrm{IC}}$ demonstrates, for the first time, that the entire anterior leaflet surface mirrors this same shape. These findings support the evolving understanding that annular shape directly influences leaflet geometry and that annuloplasty ring shape might be an important factor in maintaining leaflet curvature and reducing valvular stress after surgical repair.

The results also demonstrate that although Gaussian curvature across most of the mitral valve surface is remarkably constant, there is a single focus of regional curvature heterogeneity within the P1 region and 2 large foci in P2. This spatially dense heterogeneity can also be appreciated visually in Figure 6. We found this result to be extremely interesting for several reasons.

The most common cause of MR in patients with myxomatous leaflet degeneration is a flail midposterior (P2) leaflet caused by rupture, elongation, or both of the associated chordae tendinae. ${ }^{27}$ In general, these patients have been found to have grossly normal valvular geometry but pervasively abnormal leaflet tissue. ${ }^{28}$ Load testing of diseased valvular tissue has demonstrated mechanical failure at $50 \%$ of the load tolerated by histologically normal tissue. ${ }^{29}$ Material fatigue or degeneration involves a dynamic interface between mechanical loading, material properties, and intrinsic geometry. Assuming that all mitral leaflet tissue is subjected to a similar mechanical load and that material properties are similar throughout the mitral leaflets, it follows that the predilection for $\mathrm{P} 2$ rupture might be determined by normal patterns of regional leaflet geometry, which tends to maximize stress in this region of the valve.

Recent computed tomography-based geometric modeling of the abdominal aorta has suggested that spatially dense curvature heterogeneity and sign inversion, both of which are prominent within the $\mathrm{P} 2$ leaflet region, represent regions of high stress. ${ }^{16}$ The complex geometric relationships observed within the P2 segment of the posterior leaflet might underlie the focal leaflet fatigue and chordal rupture observed in patients with myxomatous degeneration.

In the absence of more refined finite element modeling techniques, a quantitative description of posterior leaflet geometry provides useful insight into the geometric patterns that might predispose this region to rupture. Gaussian curvature and curvature heterogeneity serve as useful parameters in this regard because the former both describes patterns of regional leaflet distortion and allows for a quantitative treatment of global leaflet shape and the latter serves as a potential surrogate for focally high stress.

The primary goal of this work was to develop a clinically applicable quantitative methodology for describing systolic mitral valve geometry in vivo. The analysis of 10 healthy human subjects presented here illustrates the utility of this technique in providing mechanistic insights into previously unexplained clinical phenomena. Although the number of subjects studied is relatively small, it is quite interesting that the shape of the valve leaflets was very similar in all 10 individuals (Figure 7). The only regions that varied substantially between valves were the P2 segment and a very small portion of the anterior leaflet adjacent to the annulus near its region of continuity with the aortic root. As previously stated, the $\mathrm{P} 2$ region demonstrated spatially dense curvature variability; interestingly, the patterns of curvature themselves also varied between individuals. The intersubject curvature heterogeneity observed in the region adjacent to the aortic root might be the result of differences in aortomitral anatomic and physiologic coupling. ${ }^{30}$ A more complete description of both of these regions will require further study.

Echocardiographic technology has improved dramatically in recent years, particularly so with the introduction of realtime 3-dimensional echocardiography. Currently, when coupled with the appropriate postprocessing software, this imaging modality can be used to provide dynamic quantitative imaging of the mitral valve with a 3-dimensional spatial resolution and registration previously unachievable. We believe strongly that because the quantitative imaging techniques presented here evolve and are applied to increasing numbers of patients, we will be able to develop predictive modeling software that will allow surgeons both to optimize repair techniques and to develop annuloplasty ring designs based, for the first time, on quantitative data rather than surgeon intuition. Ultimately, preoperative images can be used in conjunction with such software to "test" various leaflet resection and annuloplasty options to more precisely plan surgical strategies before the patient enters the operating room.

\section{References}

1. Braunberger E, Deloche A, Berrebi A, et al. Very long-term results (more than 20 years) of valve repair with Carpentier's techniques in 
nonrheumatic mitral valve insufficiency. Circulation. 2001;104(suppl 1):I8-11.

2. Chauvaud S, Fuzellier JF, Berrebi A, Deloche A, Fabiani JN, Carpentier A. Long-term (29 years) results of reconstructive surgery in rheumatic mitral valve insufficiency. Circulation. 2001;104(suppl 1): I12-5.

3. Cohn LH, Couper GS, Aranki SF, Rizzo RJ, Adams DH, Collins JJ Jr. The long-term results of mitral valve reconstruction for the "floppy" valve. J Card Surg. 1994;9(suppl):278-81.

4. David TE, Armstrong S, Sun Z, Daniel L. Late results of mitral valve repair for mitral regurgitation due to degenerative disease. Ann Thorac Surg. 1993;56:7-12.

5. Gillinov AM, Cosgrove DM 3rd, Shiota T, et al. Cosgrove-Edwards Annuloplasty System: midterm results. Ann Thorac Surg. 2000;69: 717-21.

6. Flameng W, Herijgers P, Bogaerts K. Recurrence of mitral valve regurgitation after mitral valve repair in degenerative valve disease. Circulation. 2003;107:1609-13.

7. Accola KD, Scott ML, Thompson PA, Palmer GJ 3rd, Sand ME, Ebra G. Midterm outcomes using the physio ring in mitral valve reconstruction: experience in 492 patients. Ann Thorac Surg. 2005;79:1276-83.

8. Flameng W, Meuris B, Herijgers P, Herregods MC. Durability of Mitral Valve Repair in Barlow Disease Versus Fibroelastic Deficiency. J Thorac Cardiovasc Surg. 2008;135:274-82.

9. Kim HJ, Ahn SJ, Park SW, et al. Cardiopulmonary exercise testing before and one year after mitral valve repair for severe mitral regurgitation. Am J Cardiol. 2003;93:1187-9.

10. Filsoufi F, Salzber SP, Adams DH. Current management of ischemic mitral regurgitation. Mt Sinai J Med. 2005;72:105-15.

11. Ling LH, Enriquez-Sarano M, Seward JB, et al. Early surgery in patients with mitral regurgitation due to flail leaflets: a long-term outcome study. Circulation. 1997;96:1819-25.

12. Carbello BA. Indications for mitral valve surgery. J Cardiovasc Surg. 2004:45:407-18.

13. Gillinov AM, Cosgrove DM, Blackstone EH, et al. Durability of mitral valve repair for degenerative disease. J Thorac Cardiovasc Surg. 2004; 128:916-24

14. Arts T, Meerbaum S, Reneman R, et al. Stresses in the closed mitral valve: a model study. J Biomech. 1983;16:539-47.

15. Watanabe N, Ogasawara Y, Yamaura Y, et al. Quantitation of mitral valve tenting in ischemic mitral regurgitation by transthoracic realtime three-dimensional echocardiography. J Am Coll Cardiol. 2005; 45:763-9.
16. Sacks MS, Vorp DA, Raghavan ML, Federle MP, Webster MW. In vivo three-dimensional surface geometry of abdominal aortic aneurysms. Ann Biomed Eng. 1999;27:469-79.

17. Kunzelman KS, Cochran RP, Chuong C, Ring WS, Verrier ED, Eberhart RD. Finite element analysis of the mitral valve. J Heart Valve Dis. 1993;2:326-40.

18. Kunzelman KS, Reimink MS, Cochran RP. Annular dilatation increases stress in the mitral valve and delays coaptation: a finite element computer model. J Cardiovasc Surg. 1997;5:427-34.

19. Kunzelman KS, Quick DW, Cochran RP. Altered collagen concentration in mitral valve leaflets: biochemical and finite element analysis. Ann Thorac Surg. 1998;66(suppl):S198-205.

20. Chiechi MA, Lees WM, Thompson R. Functional anatomy of the normal mitral valve. J Thorac Surg. 1956;32:378-98.

21. Gorman JH 3rd, Gorman RC, Jackson BM, et al. Distortions of the mitral valve in acute mitral regurgitation. Ann Thorac Surg. 1997;64:1026-31.

22. Tibayan FA, Rodriguez F, Langer F, et al. Increases in mitral leaflet radii of curvature with chronic ischemic mitral regurgitation. $J$ Heart Valve Dis. 2004;13:772-8.

23. Rodriguez F, Langer F, Harrington KB, et al. Effect of cutting secondorder chordae on in-vivo anterior mitral leaflet compound curvature. J Heart Valve Dis. 2005;14:592-602.

24. Sakamoto H, Parish LM, Hamamoto H, et al. Effects of hemodynamic alterations on anterior mitral leaflet curvature during systole. $J$ Thorac Cardiovasc Surg. 2006;132:1414-9.

25. Salgo IS, Gorman JH Gorman RC III, et al. Effect of annular shape on leaflet curvature in reducing mitral leaflet stress. Circulation. 2002; 106:711-7.

26. Levine RA, Handschumacher MD, Sanfilippo AJ, et al. Threedimensional echocardiographic reconstruction of the mitral valve, with implications for the diagnosis of mitral valve prolapse. Circulation. 1989;180:589-98.

27. Olson LJ, Subramanian R, Ackermann DM, Orszulak TA, Edwards WD. Surgical pathology of the mitral valve: a study of 712 cases spanning 21 years. Mayo Clin Proc. 1987;62:22-34.

28. Fornes P, Heudes D, Fuzellier JF, Tixier D, Bruneval P, Carpentier A. Correlation between clinical and histologic patterns of degenerative mitral valve insufficiency: a histomorphometric study of 130 excised segments. Cardiovasc Pathol. 1999;8:81-92.

29. Barber JE, Ratcliffe NB, Cosgrove DM, Griffin BP, Vesely I. Myxomatous mitral valve chordae. I: Mechanical properties. J Heart Valve Dis. 2001;10:320-4.

30. Timek TA, Green GR, Tibayan FA, et al. Aorto-mitral annular dynamics. Ann Thorac Surg. 2003;76:1944-50. 